\title{
Predicting Core Level Binding Energies Shifts: Suitability of the Projector Augmented Wave Approach as Implemented in VASP
}

\author{
Noèlia Pueyo Bellafont, ${ }^{1}$ Francesc Viñes, ${ }^{1, *}$ Wolfgang Hieringer, ${ }^{2,3}$ and Francesc Illas ${ }^{1}$ \\ Correspondence to: Francesc Viñes (E-mail: francesc.vines@ub.edu)
}

\author{
${ }^{1}$ Noèlia Pueyo Bellafont, Francesc Viñes, Francesc Illas \\ Departament de Ciència de Materials i Química Física \& Institut de Química Teòrica i Computacional (IQTCUB), \\ Universitat de Barcelona, C/Martí i Franquès 1, Barcelona, Spain, 08028. \\ ${ }^{2}$ Wolfgang Hieringer \\ Lehrstuhl für Theoretische Chemie, Universität Erlangen-Nürnberg, Egerlandstraße 3, Erlangen, Germany, 91058. \\ ${ }^{3}$ Wolfgang Hieringer \\ Interdisciplinary Center for Molecular Materials (ICMM), Universität Erlangen-Nürnberg, Henkestraße 42, Erlangen, \\ Germany, 91054.
}

\begin{abstract}
Here we assess the accuracy of various approaches implemented in VASP code to estimate core-level binding energy shifts ( $\triangle \mathrm{BES}$ ) using projector augmented wave (PAW) method to treat core electrons. Performance of Perdew-Burke-Ernzerhof (PBE) and Tao-Perdew-Staroverov-Scuseria (TPSS) exchange-correlation density functionals are examined on a dataset of 68 molecules containing $B \rightarrow F$ atoms in diverse chemical environments, accounting for 185 different $1 s$ core level binding energy shifts, for which experimental gas-phase X-ray photoemission (XPS) data and accurate all electron $\triangle B E s$ as well are available. Four procedures to calculate core-level shifts are investigated. JanakSlater transition state approach yields mean absolute errors of 0.37 (0.21) eV at PBE (TPSS) level, similar to highly accurate all electron $\triangle$ SCF approaches using same functionals, and close to XPS experimental accuracy of $0.1 \mathrm{eV}$. Study supports the use of these procedures to assign $\Delta \mathrm{BEs}$ of molecular moieties on material surfaces of interest in surface science, nanotechnology, and heterogeneous catalysis.
\end{abstract}

\section{Introduction}

X-ray photoelectrons spectroscopy (XPS) a.k.a. electron spectroscopy for chemical analysis (ESCA) - is nowadays a widespread technique used for material bulk elemental analysis, especially in surface science studies, given its remarkable surface sensitivity. ${ }^{1}$ Indeed, it is not only sensitive to a particular material surface termination, but to the atoms and molecules adsorbed onto, which makes it a well-suited technique to detect surface adsorption conformations., ${ }^{3,4}$ The detection of emerged electrons over time allows to in situ follow a given surface process, from adsorption and diffusion, to surface reactions heterogeneously catalyzed by the substrate, enabling the characterization of reactants, intermediates, and products, and, ultimately, getting information of the reaction mechanism and kinetics. ${ }^{2,5,6}$

The discrimination of one from another species (or conformations) links to particular atomic core level (CL) binding energies and, more importantly, core level binding energy 
shifts with respect to a given reference, which provide information about different chemical environments and/or oxidation states. The development of high resolution XPS (HR-XPS) technique allows nowadays to distinguish core binding energy shifts $(\triangle \mathrm{BE})$ to a precision of up to $\sim 0.1 \mathrm{eV} .{ }^{6}$ However, the definite correlation of one observed XPS peak to a given atom within a molecule and to its chemical environment is by no means straightforward. Usual procedures are based upon gas phase molecular data, ${ }^{7}$ or well-defined surface science experiments under very controlled conditions. ${ }^{2,6}$ A more solid, unbiased identification via accurate calculations simulating the electron ionization process would be very attractive. Along this line wave function and density functional theory (DFT) based methods have proven its suitability using the so-called $\triangle S C F$ approach, ${ }^{8}$ in which the binding energy (BE) for a given atom $\mathrm{CL}$ is gained by the difference in total energy between the neutral system, and that with an hole in the $\mathrm{CL}$ of the atom under inspection.

The BEs values obtained through the $\triangle \mathrm{SCF}$ approach are obtained at the final state -i.e. the excited core electron is removed from the system under scrutiny and the electronic structure modified according to the electron hole. Indeed, by considering the electronic screening of the core state and relativistic effects, one can obtain BE estimates well within the XPS experimental accuracy of $0.1 \mathrm{eV}$, either based on Hartree-Fock (HF) calculations, or using methods within DFT. ${ }^{9,10}$ Such a high level of accuracy for isolated molecules would be desirable when they are adsorbed to a solid surface, in order to distinguish among different molecular adsorption conformations, as well as to monitor heterogeneously catalyzed processes.

Specifically, it is highly desirable to correctly model adatoms and adsorbed molecules on transition metal (TM) surfaces, given that they are ubiquitous in heterogeneous catalysis as supported active phases. One has also to keep in mind that these systems play a key role in other fields as well such as nanotechnology devices and chemical resolution components. A recent systematic study identified the PerdewBurke-Ernzerhof $(\mathrm{PBE})^{11}$ and the Tao-PerdewStaroverov-Scuseria (TPSS) ${ }^{12}$ DFT exchangecorrelation functionals as best compromises suited to simultaneously describe both main group molecules and TM surfaces. ${ }^{13,14}$ One of the most extended ways of tackling $a b$ initio studies of TM surfaces is to take advantage of periodic boundary conditions, ${ }^{15}$ and, especially for TM, to use pseudopotentials to describe core electrons or the projector augmented wave (PAW) method, ${ }^{16}$ being highly accurate and in extensive use. This formalism can be regarded as an effective all electron method in which atomic cores are frozen as in the isolated atom in its spin polarized ground state. In the case one is interested in core level binding energies, the PAW description of the atomic core with a generated core hole can be employed. ${ }^{17}$ Several implementations of the PAW method exist in the literature with different specificities. Here we focus in the implementation of Kresse of Joubert ${ }^{18}$ in the Vienna $a b$ initio simulation package (VASP) code. ${ }^{19}$ This is a code specially designed to study periodic systems yet also suited to finite systems provided they are embedded in a periodic box sufficiently large to effectively isolate the contained species. ${ }^{20}$

However, periodic slab models, despite allowing for a reasonable description of surface processes on TM surfaces, hamper a rigorous application of the $\triangle$ SCF approach. This is because of two different reasons; i) the resulting system with the core hole is periodically charged and ii) the core hole use to be simulated with a pseudopotential rather than the full electronic description of core electrons. Despite of these difficulties, it has been shown that final state (FS) CL BEs can be estimated by generating a core excited ionic PAW in the course of the corresponding calculation, as above commented. ${ }^{17}$ The approach neglects relaxation of core electrons upon ionization, yet valence electron screening is explicitly described. In this approach, to avoid dealing with a periodically repeated charged system, the excited electron is added to the 
bottom of the former conduction band - or the lowest unoccupied molecular orbital (LUMO) in the case of isolated molecules-, see Figure 1. The system thus maintains its charge neutrality within the imposed periodic boundary conditions (PBC). While this approach seems appropriate to describe XPS with conducting probes, for isolated molecules a naïve application of the same approach would result in actually modeling a X-ray absorption near edge structure (XANES) spectroscopy process. Notice that within the VASP implementation of the PAW method, absolute BEs are not accessible given that only valence energies are calculated. Other implementations exist where absolute $\mathrm{BE}$ can be obtained by adding up the frozen core energies, ${ }^{21}$ although so far its use has been limited. Because of the limitation in performing proper $\triangle \mathrm{SCF}$ in the employed code, we here focus on core level binding energy shifts $(\triangle \mathrm{BE})$, alleged however to be rather accurate $^{17}$ and widely used in practice to assign XPS signatures to certain surface species..$^{3-5,22-24}$

The precision of this particular FS approach has been estimated to be of $0.02-0.05 \mathrm{eV}$ in test calculations on selected systems. ${ }^{17}$ Subsequent studies reported larger deviations, ${ }^{3-5,24}$ and, in practice, different approaches are frequently considered, seeking for the best applied approach, as the accuracy issue remained unclear. Other approaches include initial state (IS) approximations, which considers that KohnSham (KS) eigenstate energies in the electronic ground state as approximations to BE - similar to Koopmans theorem in Hartree-Fock, a statement recently discussed unambiguously showing that while Koopmans theorem does not hold for the KS eigenvalues, it properly reflects the trends in $\triangle \mathrm{BEs} .{ }^{25}$ The proper way to define IS BEs from DFT calculations has also been described by some of us. ${ }^{26}$ An alternative approach is provided by the model known as Janak-Slater (JS) transition state method, ${ }^{27}$ which, in a nutshell, considers half occupation of the $C L$ when estimating $B E s$ rather than a full core-hole as in the FS approach, see Figure $1 .{ }^{28}$ Here, the orbital energies are used as estimates for $\mathrm{CL} B E s$ and total energies are actually disregarded.

To address the accuracy of the these procedures above described, here we take advantage of the recent evaluation of PBE and TPSS performance on estimating $\triangle$ SCF BES and $\triangle \mathrm{BEs}$ for $1 \mathrm{~s} \mathrm{CL}$ for a large data set of 68 molecules containing a wide variety of functional groups for first row main group elements $B \rightarrow F$, considering up to 185 core levels, for which accurate gas phase experimental BEs are known, see Table S1 in supplementary information (SI). ${ }^{9,29-32}$ Only these functionals have been assessed, since the ultimate goal is to employ them to assign XPS features of atoms and/or molecules in different positions and/or conformations on TM supports. The $\triangle \mathrm{SCF}$ calculations for this large set of molecules have been previously carried out using a full all electron description and a near Hartree-Fock limit basis set, obtained within spin restricted open-shell formalisms for both HF and KS methods. ${ }^{9}$

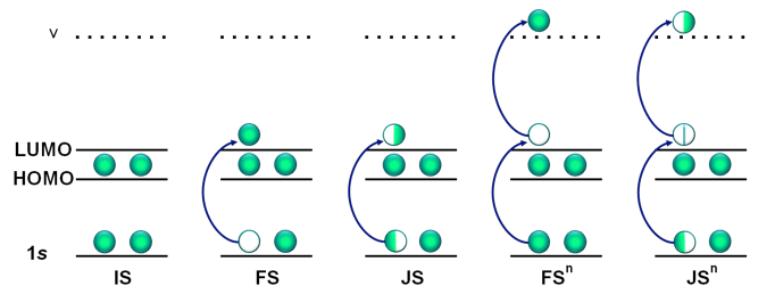

Figure 1. Sketch of initial state (IS), final state (FS), and Janak-Slater (JS) situations, as well as $\mathrm{FS}^{\mathrm{n}}$ and $\mathrm{JS}^{\mathrm{n}}$ approaches where excited (half) electron is removed from the system, and conceptually placed in vacuum, $v$.

\section{Computational Details}

In the present calculations data set molecules were isolated in an asymmetric box with PBC ensuring a minimum vacuum distance in any direction of $10 \AA$. Calculations were carried out at the $\Gamma$-point and using a cut-off kinetic energy limit of 415 for the plane-wave basis set. The PAW approach was used to treat core electrons and PAW core orbital energies were used to deduce the BE shifts in all cases. An electronic 
convergence $0.01 \mathrm{eV} \AA^{-1}$ were used, together to a $1^{\text {st }}$ order Methfessel-Paxton smearing of 0.1 $\mathrm{eV}$ width. Calculations were performed in a spin-polarized and a non spin-polarized fashion, although criterion of $10^{-6} \mathrm{eV}$ and an ionic convergence force criterion of spin-polarization effect on BEs was found to be essentially negligible, and so for the $\triangle \mathrm{BEs}$.

Note that spin polarization is a necessity to properly estimate absolute BEs, either using $\mathrm{HF}$ or KS formalisms. Indeed, its neglecting by carrying out spin restricted HF or KS in which the core hole is evenly distributed among $\alpha$ and $\beta$ spin-orbitals, yields deviations of core orbital energies of up to $\sim 10 \mathrm{eV},{ }^{21}$ an error remediated by carrying out calculations either spin unrestricted or open-shell. Despite of this, even if $\mathrm{BE}$ values obtained from $\triangle \mathrm{SCF}$ calculations may be affected by spin polarization effects, there is evidence that the effects on the $\triangle B E s$ are negligible. ${ }^{33}$ Consequently, only non spinpolarized values are discussed in the following. All calculations have been carried out using the VASP program. ${ }^{19}$

The IS, FS, and JS BEs and the corresponding shifts, $\triangle B E$, with respect to a reference have been obtained following the previously prescribed methodology, with the following modifications: i) BEs are referred to the energy of an electron in vacuum (instead of the Fermi energy, $\left.E_{F}\right) .{ }^{24}$ Vacuum energy level is obtained in each computed case as the constant value electrostatic potential energy distant from the molecule. Note that this vacuum leveling has been carried out independently for each molecule, studied 1s level, and approximation (IS, JS, or FS based). Next, ii) for cases involving highly-symmetry related cores different PAW types were used for these equivalent atoms to avoid delocalized electronic situations. In such cases the PAW used for the atom under evaluation was kept as the standard in use, but the PAW of the other atom(s) related by symmetry replaced by the PAW_h references. ${ }^{18}$ Finally iii) for FS and JS, instead of the usual addition of the excited electron - or half electron in the JS case - to the LUMO, a more physically sound situation has been contemplated, in which the excited (half) electron is removed from the system, hereafter named $\mathrm{FS}^{\mathrm{n}}$ and $J \mathrm{~S}^{\mathrm{n}}$ methods, see Figure 1 . The resulting charge is compensated inside the PBC box with an underlying countercharge background distributed within the box. The $J S^{n}$ approach thus closely corresponds to JanakSlater transition state approaches within periodic boundary conditions. Furthermore, by comparison, this approach allows estimating the artificial screening effect of (half) electrons in the LUMO in the FS and JS approaches. The possible effect on $\triangle B E s$ of the box-diluted countercharge has been evaluated by doubling the cell dimensions, i.e. minimizing the background countercharge density, with negligible variations $-\sim 0.03 \mathrm{eV}$.

\section{Results}

The BEs obtained using the JS, FS, JS ${ }^{n}$, and $\mathrm{FS}^{\mathrm{n}}$ protocols on PBE and TPSS density functionals are listed in Table S1 in SI, whereas those obtained at IS are listed in Table S2. The $\triangle$ SCF approach using PAW DFT total energy values (not shown) give very discrepant results with respect to experiments, since, as commented, only valence electron density energy is used when core electrons are described by the PAW pseudopotentials. However, IS BE values (i.e., ground-state core orbital energies), despite being heavily and unphysically underestimated, capture the experimental BE trends (see Figure S1 in SI), as expected from previous works. ${ }^{25}$ The shift with respect to experiment, estimated according to mean error (ME) and mean absolute error (MAE), shown in Table S3 in SI, backs up an arbitrary shifting procedure sometimes used when attempting at comparing these calculated values with experimental measurements. ${ }^{17}$

The underestimation of absolute binding energies by the ground-state core orbital energies in the IS approach -implying a negative relaxation energy in response to the presence of the core hole- flips to overestimation when applying any of the final state approaches $-\mathrm{FS}, \mathrm{JS}, \mathrm{FS}^{n}$, or $\mathrm{JS}^{n}-$, as is 
exemplified for $\mathrm{C} 1 \mathrm{~s}$ in Figure S2 of SI. It is worth to highlight that overall $i)$ PBE values are closer to experimental ones than TPSS and that ii) FS and JS values are much closer to experimental values than the $\mathrm{FS}^{\mathrm{n}}$ and $\mathrm{JS}^{\mathrm{n}}$ counterparts, yet iii) the trend is better captured using $\mathrm{FS}^{\mathrm{n}}$ and $\mathrm{JS}^{\mathrm{n}}$ approaches, and iv) JS and $J S^{n}$ values are much closer to experimental values than $\mathrm{FS}$ an $\mathrm{FS}^{\mathrm{n}}$. As in IS, MAE, see Table S4, indicates the presence of a systematic deviation which allows for shifting $B E$ values to align them with the experimental ones.

The not so unexpected inaccuracy of absolute BEs, which can be, in the best cases, of up to few eV, cancels however when estimating core level binding energy shifts. To illustrate this point, $\triangle B E s$ were separately gained for main group elements $B \rightarrow F$ with respect to a given simple reference molecule: diborane $\left(\mathrm{B}_{2} \mathrm{H}_{6}\right)$, methane $\left(\mathrm{CH}_{4}\right)$, ammonia $\left(\mathrm{NH}_{3}\right)$, water $\left(\mathrm{H}_{2} \mathrm{O}\right)$, and fluoromethane $\left(\mathrm{CH}_{3} \mathrm{~F}\right)$ for $\mathrm{B} \rightarrow \mathrm{F}$, respectively. A comparison of the performance for $\mathrm{FS}, \mathrm{JS}, \mathrm{FS}^{\mathrm{n}}$, and $\mathrm{JS}^{\mathrm{n}}$ in calculating $\triangle \mathrm{BEs}$ versus experimental values is provided in Figure 2.

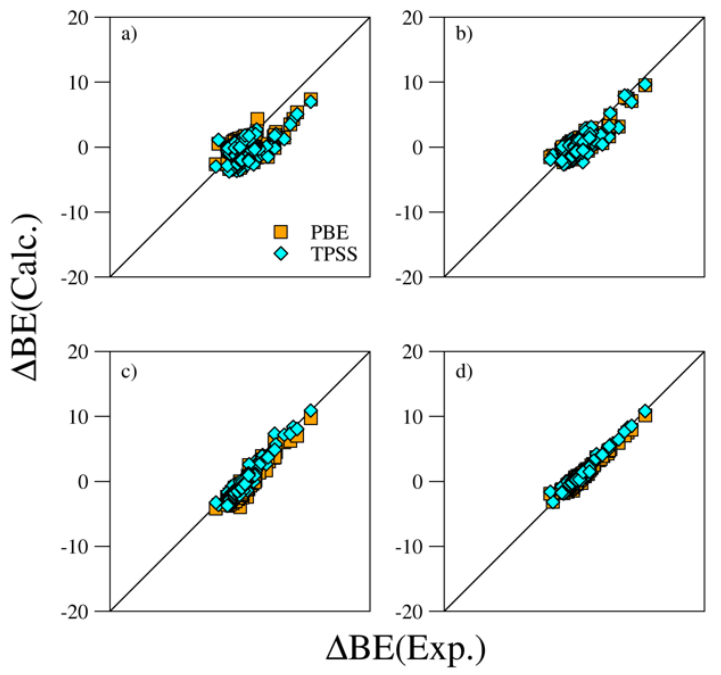

Figure 2. Calculated PBE and TPSS $\triangle B E S$, $\triangle B E($ Calc.) versus experimental values, $\triangle B E\left(\operatorname{Exp}\right.$.), for a) FS, b) JS, c) $F S^{n}$, and d) $J S^{n}$ methods. All values are given in $\mathrm{eV}$.

Note, for instance, how FS values tend to be underestimated at both PBE and TPSS levels, with PBE values slightly closer to experimental values, although with a clear wider dispersion. When applying the JS approach, values walk the line, and so the MAE decreases, see Table 1, although the slightly better performance of PBE versus TPSS remains, though the underestimation seems to vanish. However, the MAE values for PBE and TPSS within the FS and JS approaches are $0.9 \mathrm{eV}$ and $1.0 \mathrm{eV}$, respectively, and so, of little use when trying to distinguish species/conformations within the aforementioned experimental precision of 0.1 eV.

The situation is greatly improved when removing the excited (half) electron from the system, e.g. when carrying out $\mathrm{FS}^{n}$ and $\mathrm{JS}^{n}$ approximations. The removal of the ejected electron clearly lines up $\triangle B E s$ estimates either at full core-hole $\left(\mathrm{FS}^{\mathrm{n}}\right)$ or within the Janak-Slater $\left(J S^{n}\right)$ approximation. However, deviations are still noticeable in the $\mathrm{FS}^{\mathrm{n}}$ case, especially at PBE level, with an underestimation of $-1.4 \mathrm{eV}$, which drops to $0.85 \mathrm{eV}$ at TPSS level. Notice how the artificial valence (half) electron screening by PAW CL estimation increased errors by 0.19 and $0.80 \mathrm{eV}$ at PBE and TPSS levels, respectively, as observed by comparing $\mathrm{FS}$ and $\mathrm{FS}^{\mathrm{n}}$ values.

\begin{tabular}{|c|c|c|c|c|}
\hline & \multicolumn{4}{|c|}{ PBE } \\
\hline & FS & JS & $\mathrm{FS}^{\mathrm{n}}$ & $J S^{n}$ \\
\hline $\mathrm{ME}$ & -1.03 & -0.28 & -1.40 & -0.21 \\
\hline \multirow[t]{3}{*}{ MAE } & 1.62 & 0.90 & 1.43 & 0.37 \\
\hline & \multicolumn{4}{|c|}{ TPSS } \\
\hline & FS & JS & $\mathrm{FS}^{\mathrm{n}}$ & $J S^{n}$ \\
\hline $\mathrm{ME}$ & -1.21 & -0.42 & -0.85 & 0.02 \\
\hline MAE & 1.73 & 1.01 & 0.93 & 0.21 \\
\hline
\end{tabular}

However the most important result is that the half excited electron removal in $J S^{n}$ not only lines up estimates, but also deviations. As a result, no clear underestimation can be discerned, and, moreover, MAE values diminish to 0.37 and $0.21 \mathrm{eV}$ for PBE and TPSS JS ${ }^{\mathrm{n}}$ levels. As found for $\mathrm{FS}^{\mathrm{n}}$, the half excited electron screening was responsible of an average increase of errors in $\triangle B E$ s of 0.53 and $0.8 \mathrm{eV}$ for 
PBE and TPSS, respectively. More importantly, the $\triangle B E S$ MAE at PBE and TPSS levels of 0.37 and $0.21 \mathrm{eV}$ are of the same accuracy of corresponding relativistic $\triangle S C F$ calculations of 0.24 and $0.25 \mathrm{eV}$ as obtained in an earlier work. ${ }^{9}$ Hence, for gas phase molecules, the $\mathrm{JS}^{\mathrm{n}}$ method in combination with a PAW core description is capable to reach the same high degree of accuracy for $\triangle B E s$ than all electron $\triangle S C F$ using a fully uncontracted near HartreeFock limit basis set. ${ }^{9}$ Note that for thermochemistry of gas phase molecules hybrid functionals, such as Becke-Lee-Yang-Parr (B3LYP), are slightly better than PBE or TPSS, with a MAE of $0.16 \mathrm{eV}$, as obtained from relativistic $\triangle S C F$ calculations on a subset of data, ${ }^{10}$ although its use is not advised for treating TM systems, as the proper metallic bond delocalization is hindered when using hybrids. ${ }^{14}$ Last but not least, one has to keep in mind that small, yet frequent, experimental BE shifts which fall within the PBE and TPSS JS ${ }^{n}$ accuracies cannot be correctly backed up by such calculations, and improved accuracy methods should be used, as the above commented. In any case, TPSS seems to be the best compromise and better suited when trying to link experimental $\triangle \mathrm{BEs}$ to $a b$ initio estimations, both reaching a similar degree of precision. Note that the superior performance of the $\mathrm{JS}^{\mathrm{n}}$ method (used with an PAW core description and periodic boundary conditions here) may not be surprising based on its analogy to the original Slater transition state theory. $^{28}$

\section{Conclusions}

In conclusion, we addressed the accuracy of full core-hole (FS) and Janak-Slater (JS) approaches as implemented in VASP in predicting core level binding energies and, more specifically, core level binding energy shifts from core orbital energies using a PAW description of the atomic cores, and under periodic boundary conditions, on a broad dataset of 68 molecules containing first row atoms $(B \rightarrow F)$ in a great diversity of chemical environments, accounting for a total number of 185 different 1 s CLs. In addition to current procedures we contemplated removing the excited (half) electron from the molecular LUMO in the here called FS ${ }^{n}$ and $\mathrm{JS}^{\mathrm{n}}$ approaches. As expected, the PAW total BE estimates predicted by the VASP code do not match $\mathrm{CL}$ BEs since core electron energies are not explicitly accounted for. However, computed $\triangle B E s$ estimates, i.e. relative $B E$ shifts, line up well with experimental trends. For PBE and TPSS exchange-correlation functionals $\triangle B E$ estimates using $\mathrm{JS}^{\mathrm{n}}$ approach yield mean absolute errors of only 0.37 and $0.21 \mathrm{eV}$, and are thus of similar size to all electron $\triangle S C F$ calculations with saturated basis sets, ${ }^{9}$ thereby approaching the experimental accuracy of HRXPS of $0.1 \mathrm{eV}$. The accuracy of the $\mathrm{JS}^{\mathrm{n}}$ approach is useful not only for molecules, but also promises its use in assigning $\triangle B E s$ of molecular moieties on material surfaces, with concomitant applications in surface science, nanotechnology, and heterogeneous catalysis, although its accuracy in such systems remains to be confirmed.

\section{Acknowledgments}

This work has been supported by Spanish MINECO/FEDER CTQ2015-64618-R grant and, in part, by Generalitat de Catalunya grants 2014SGR97 and XRQTC, and by the NOMAD Center of Excellence project, which received funding from the European Union's Horizon 2020 research and innovation programme under grant agreement No 676580. F.V. thanks MINECO for a postdoctoral Ramón y Cajal (RyC) research contract (RYC-2012-10129). F.I. acknowledges additional support from the 2015 ICREA Academia Award for Excellence in University Research. W.H. gratefully acknowledges support from the Deutsche Forschungsgemeinschaft through the "Cluster of Excellence - Engineering of Advanced Materials" located in Erlangen, Germany.

Keywords: XPS - Core level binding energy shifts - PAW · DFT · Molecular dataset 
Additional Supplementary Information (SI) can be found in the online version of the article: Molecular list with BE estimates at both PBE and TPSS under FS, JS, FS ${ }^{n}$, and $J^{n}{ }^{n}$ approaches is given in Table S1. The IS and core orbital energy estimates are listed in Table S2. A statistical analysis of BEs is shown in Tables S3 and S4. Element decomposed IS trends are shown in Figure S1, whereas FS, JS, FS ${ }^{n}$, and $\mathrm{JS}^{\mathrm{n}}$ BE trends for C1s cases are shown in Figure S2.

\section{References and Notes}

1. K. Siegbahn; C. Nordling; A. Falhman; R. Nordberg; K. Hamrin; J. Hedman; G. Johansson; T. Bergmark; S. E. Karlsson; I. Lindgren; B. Lindberg in ESCA (Atomic, Molecular, and Solid State Structure Studied by Means of Electron Spectroscopy; Alwmvist and Wiksells Boktryckeri AB, Uppsala, Sweden, 1967.

2. M. Salmeron; R. Schlögl, Surf. Sci. Rep. 2008, 63, 169.

3. K. Gotterbarm; N. Luckas; O Höfert; M. P. A. Lorenz; R. Streber; C. Papp; F. Viñes; H. P. Steinrück; A. Görling, J. Chem. Phys. 2012, 136, 094702.

4. W. Zhao; S. M. Kozlov; O Höfert; K. Gotterbarm; M. P. A. Lorenz; F. Viñes; C. Papp; A. Görling; H. P. Steinrück, J. Phys. Chem. Lett. 2011, 2, 759.

5. N. Luckas; K. Gotterbarm; R. Streber; M. P. A. Lorenz; O. Höfert; F. Viñes; C. Papp; A. Görling; H. P. Steinrück, Phys. Chem. Chem. Phys. 2011, 13, 16227.

6. C. Papp; H. P. Steinrück, Surf. Sci. Rep. 2013, 68, 446.

7. K. Siegbahn; C. Nordling; G. Johansson; J. Hedman; P. F. Hedén; K. Hamrin; U. Gelius; T. Bergmark; L. O. Werme; R. Manne; Y. Baer, in ESCA Applied to Free Molecules; North-Holland: Amsterdam, 1969.

8. P. S. Bagus; F. Illas; G. Pacchioni; F. Parmigiani, J. Electron Spectrosc. Relat. Phenom. 1999, 100, 215.

9. N. Pueyo Bellafont; F. Viñes; F. Illas, J. Chem. Theory Comput. 2016, 12, 324.
10. N. Pueyo Bellafont; G. Álvarez Saiz; F. Viñes; F. Illas, Theor. Chem. Acc. 2016, 135, 35.

11. J. P. Perdew; K. Burke; M. Ernzerhof, Phys. Rev. Lett. 1996, 77, 3865.

12. J. Tao; P. Perdew; V. N. Staroverov; G. E. Scuseria, Phys. Rev. Lett. 2003, 91, 146401.

13. P. Janthon; S. M. Kozlov; F. Viñes; J. Limtrakul; F. Illas, J. Chem. Theory Comput. 2013, 9, 1631.

14. P. Janthon; S. Luo, S. M. Kozlov; F. Viñes; J. Limtrakul; D.G. Truhlar, F. Illas, J. Chem. Theory Comput. 2014, 10, 3832.

15. F. Bloch, Z. Phys. 1929, 52, 555.

16. P. E. Blöchl, Phys. Rev. B 1994, 50, 17953.

17. L. Köhler; G. Kresse, Phys. Rev. B 2004, 70, 165405.

18. G. Kresse; J. Joubert, Phys. Rev. B 1999, 59, 1758.

19. G. Kresse; J. Furthmüller, Phys. Rev. B 1996, 54, 11169.

20. F. Viñes; J. R. B. Gomes; F. Illas, Chem. Soc. Rev. 2014, 43, 4922.

21. M. P. Ljungberg; J. J. Mortensen; L. G. M. Pettersson, J. Electron Spec. Rel. Phenom. 2011, 184, 427.

22. Y.-Q. Zhang; N. Kepcija; M. Kleinschrodt; K. Diller; S. Fischer; A. C. Papgeorgiou; F. Allegretti; J. Björk; S. Klyatskaya; F. Klappenberger; M. Rubén; J.V. Barth, Nat. Commun. 2012, 3, 1286.

23. R. C. Salvarezza; P. Carro, Phys. Chem. Chem. Phys. 2015, 17, 24349.

24. P. Torelli; L. Giordano; S. Benedetti; P. Luches; E. Annese; S. Valeri; G. Pacchioni, J. Phys. Chem. C 2009, 113, 19957.

25. N. Pueyo Bellafont; F. Illas; P. S. Bagus, Phys. Chem. Chem. Phys. 2015, 17, 4015.

26. N. Pueyo Bellafont; P. S. Bagus; F. Illas, J. Chem. Phys. 2015, 142, 214102.

27. J. F. Janak, Phys. Rev. B 1978, 18, 7165.

28. J. C. Slater, Adv. Quantum. Chem. 1972, 6, 1.

29. A. A. Bakke; H. W. Chen; W. L. Jolly; J. Electron Spectrosc. Relat. Phenom. 1980, $20,333$.

30. W. L. Jolly; K. D. Bomben; C. J. Eyermann, At. Data Nucl. Data Tables, 1984, 31, 433. 
31. Y. Takahata; D. P. Chong, J. Electron Spectrosc. Relat. Phenom. 2003, 133, 69.

32. V. Myrseth; L. J. Saethre; K. J. Borve; T. D. Thomas, J. Org. Chem. 2007, 72, 5715.

33. D. P. Chong, Chem. Phys. Lett. 1995, 232, 486. 
Noèlia Pueyo Bellafont, Francesc Viñes, Wolfgang Hieringer, and Francesc Illas

Predicting Core Level Binding Energies Shifts: Suitability of Projector Augmented Wave Approach as Implemented in VASP

Here we assess the accuracy of various approaches implemented in VASP to estimate core-level bindingenergy shifts ( $\triangle \mathrm{BEs})$ using projector-augmented-wave method to treat core electrons. Different exchange-correlation functionals within density functional theory are tested on a 68 molecules dataset accounting 185 different $1 s(\Delta \mathrm{BEs}$ ) for which experimental data is available. Janak-Slater transition state approach yields an accuracy similar to all electron $\triangle \mathrm{SCF}$ calculations, and close to $\mathrm{X}$-ray photoemission accuracy of $0.1 \mathrm{eV}$.

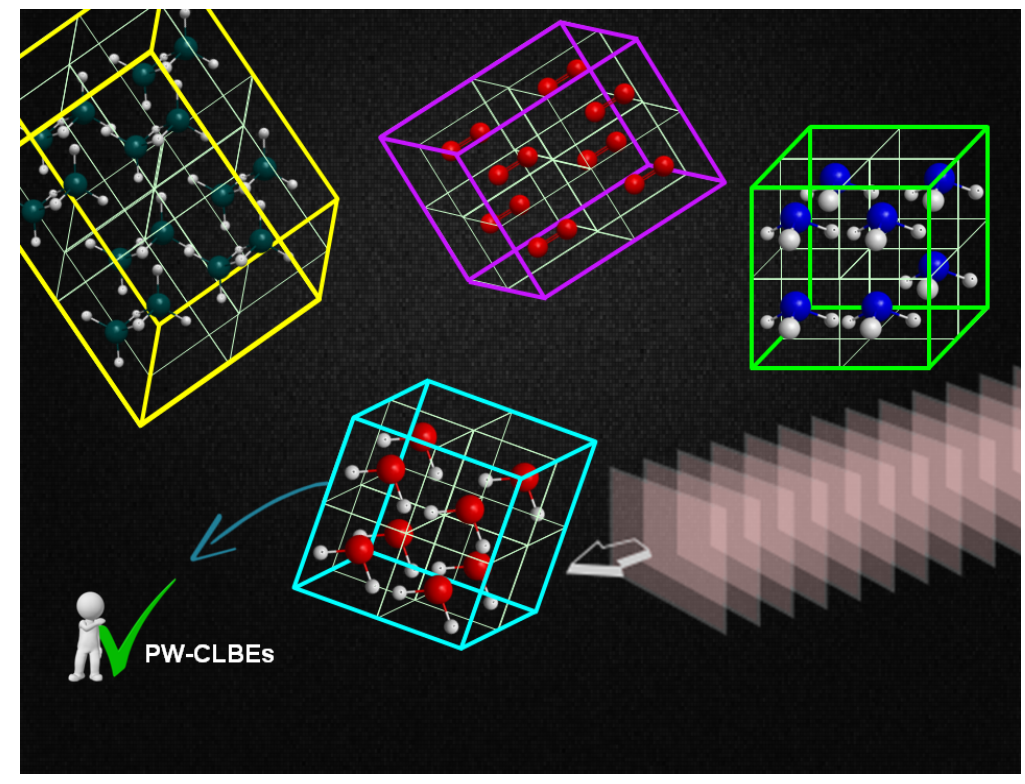

